\title{
Study of Association of Primary Vesicoureteric Reflux in Children Suffering from Urinary Tract Infection
}

\author{
Bablu kumar Saha \\ Md Mahfuzul Haque ${ }^{1^{*}}$ \\ Swapan Kumar Paul ${ }^{2}$ \\ Mohammed Shadrul Alam \\ Md Shahid Karim ${ }^{3}$ \\ ${ }^{1}$ Department of Pediatric Surgery \\ Rangpur Medical College \& Hospital \\ Rangpur, Bangladesh. \\ ${ }^{2}$ Department of Pediatric Surgery \\ Dhaka Shishu (Children) Hospital \\ Dhaka, Bangladesh.
}

${ }^{3}$ Department of Pediatric Surgery \& Pediatric Urology Apollo Hospitals Ltd Dhaka, Bangladesh.

\section{*Correspondence to:}

\section{Dr. Md. Mahfuzul Haque}

Registrar

Department of Pediatric Surgery

Rangpur Medical College \& Hospital

Rangpur, Bangladesh.

Mobile: +8801712000017

E-mail: mahfuzps@gmail.com

\begin{abstract}
Purpose: The retrograde flow of urine from the bladder to the upper urinary tract is known as vesicoureteral reflux (VUR). The association between VUR and UTI represents a significant threat to the kidney in the form of pyelonephritic scarring. Early diagnosis of reflux and identification of the pyelonephritic aggression are important for successful therapy. Purpose of this study is to find out the association between primary VUR and urinary tract infection. Objective: Early diagnosis of VUR presenting as UTI to prevent renal damage and also to asses the result of initial management of primary VUR. Materials and methods: From April 2004 to April 2005, 40 patients of documented UTI were evaluated with VCUG to demonstrate VUR in Dhaka Shishu Hospital. VUR grade III and above were further evaluated with DMSA renal scan to demonstrate renal scarring. Among reflux patients 6 patients underwent surgery (Cohen ureteric reimplantation), 8 patients were treated non-operatively and one patient died of renal failure. Non- VUR grouped patients were treated with antibiotic according to culture sensitivity report for 10 days. VUR patients were followed up for 12 weeks. Results: E.coli was the commonest organism causing UTI. Fifteen patients (38\%) having different grades of primary VUR and it was more in male child and in below 1- year age group. Episodes of fever were the predominant clinical scenario followed by failure to thrive, loss of appetite, cloudy urine and frequent voiding. ARM was the commonest associated anomaly (27\%). Conclusion: UTI especially in male child was associated with VUR in $38 \%$ of this study population. ARM was the common associated anomaly. Renal scarring was noted in higher grade of reflux.
\end{abstract}

Key words: Vesicoureteral reflux (VUR); urinary tract infection (UTI); pyelonephritis.

\section{INTRODUCTION}

In infant and children, the urinary tract is a relatively common site of infection. At least $8 \%$ of girls and $2 \%$ of boys will have UTI during childhood ${ }^{1}$. Urinary tract infections result in significant acute morbidity as well as long-term medical problems including delayed hypertension and progressive renal dysfunction. Accurate diagnosis and timely treatment are vital in limiting these long-term sequelae because the pediatric kidney is particularly susceptible to permanent renal damage ${ }^{2}$.

The retrograde flow of urine from the bladder to the upper urinary tract is known as vesicoureteral reflux $(\mathrm{VUR})^{3}$. This anomaly is considered primary when there is no demonstrable urinary out flow obstruction ${ }^{4}$.

The association between VUR and UTI represents a significant threat to the kidney in the form of pyelonephritic scarring ${ }^{3}$. In clinical practice, the association between VUR and urinary infection is well recognized; urinary infection is the major factor leading to identification of VUR and patients with VUR acquire recurrent urinary infection ${ }^{5}$. VUR is most commonly diagnosed after a urinary tract infection. Average $30 \%-50 \%$ of all children, boys and girls, show VUR when evaluated after their first urinary tract infection ${ }^{6}$. 
Although clinical signs and symptoms are important indications of childhood urinary tract infection, owing to the profound implications of UTI in children, confirmation of the diagnosis by microscopic examination and quantitive culture of a properly collected specimen is imperative. The accepted criteria for diagnosis of UTI is growth of single bacterial species $>10^{5} \mathrm{CFU} / \mathrm{ml}$ urine ${ }^{7}$.

UTI in young children serve as a marker for abnormalities of the urinary tract. Imaging of the urinary tract is recommended in every febrile infant or young children with a first UTI to identify those with abnormalities that predispose to renal damage. Imaging should consist of urinary tract ultrasonography to detect dilatation secondary to obstruction and a study to detect VUR ${ }^{8}$. Voiding cystourethrogram (VCUG) remains the technique of choice for the diagnosis and classification of VUR ${ }^{9}$. Radionuclide cystography is, however, preferred during follow up, as it affords lower radiation exposure to the gonads ${ }^{10}$.

The problem of UTI, when associated with VUR, in fact is like the tip of iceberg. The family \& physicians may be aware of the problem of micturition and treated the child with antibiotics for 7-10 days. But the actual picture of life-threatening internal damages may remain undiscovered.

\section{MATERIALS \& METHODS}

This cross-sectional study was carried out in the department of pediatric surgery, Dhaka Shishu (children) Hospital (DSH) during the period of July 2003 to July 2005.

Prior to commencement of this study the ethical review committee of Bangladesh Institute of Child health (BICH) and Dhaka Shishu (children) hospital approved the protocol. The aims and methods of the studies were explained to the legal guardians of the patients. The subjects, who agreed to participate, gave written consent. In each case, information about the patients was obtained in the form of a questionnaire after getting consent of the guardian in the preformed consent form. All the patients meeting selection criteria were enrolled consecutively. Sample size was 40.

Inclusion criteria were Patients with suspected UTI reported in surgery outpatient department, referred cases of documented UTI from Pediatrician of Dhaka Shishu Hospital and outside and Incidental finding of UTI in patients admitted with nonurological problem.

Exclusion criteria were Urine $\mathrm{C} / \mathrm{S}$ did not fulfill the criteria of documented UTI, patients did not come for follow up and Secondary VUR and VUR with exstrophy-epispadias complex.

\section{Study Procedure}

Patients were collected from Surgery Out Patient Department after evaluation of patient with suspected UTI and from pediatrician of Dhaka Shishu Hospital and outside as referred cases of documented UTI (Growth of single bacteria was $>10^{5}$ $\mathrm{CFU} / \mathrm{ml}$ of clean voided mid stream urine). The researcher filled up the questionnaire forms after consent of guardians. The questionnaire included age, weight, sex, address of the patient, yearly income, birth history, presenting complaints with duration, any relevant past history and detailed physical examination. Detailed history of micturition including its flow (poor or narrow stream, dribbling, straining), color, retention, frequency, failure to thrive, presence of flank or suprapubic mass or any other complaints were noted. The associated signs including blood pressure were also recorded after thorough physical examination especially urinary system. Clean voided mid stream urine was collected for microscopy and culturesensitivity. Antibiotic was started, which was continued or changed according to culture-sensitivity report. Patients with documented UTI then evaluated with USG of KUB region and VCUG. Patients having VUR grade III and above further evaluated with DMSA renal scan. Patients were grouped as having primary VUR and non-VUR. Primary VUR grouped patients were treated operatively and non-operatively and followed up with prophylactic antibiotics. Non-VUR grouped patients were treated with antibiotics for 10 days only. The investigations were carried out to correlate the clinical diagnosis and to determine complications of UTI including laboratory tests, radiology and imaging studies.

\section{BLOOD}

Hemoglobin percentage $(\mathrm{Hb} \%)$, Total and differential count of WBC, Blood grouping*, Blood urea, Serum creatinine and Serum electrolytes.

\section{URINE}

Routine and microscopic examination $(\mathrm{R} / \mathrm{M} / \mathrm{E})$, Culture and sensitivity test $(\mathrm{C} / \mathrm{S})$.

\section{RADIOLOGY AND IMAGING}

Ultrasonogram of the kidney ureter and bladder (KUB), Voiding cystourethrogram (VCUG) and Isotope Reno gram (DMSA) ** (* Where blood transfusion may be required.** Isotope Reno gram was done in patients with VUR grade III and above)

Confirmation of UTI and subsequent VCUG, patients were grouped as VUR and Non-VUR. VUR grouped patients were treated either operatively or non-operatively and followed up by giving advice to take prophylactic antibiotic, perineal hygiene, taking adequate water, regular bowel habit and to report at the surgical out patient department for follow-up. Operative treatment was done in all bilateral grade IV, grade V and grade III with single kidney. Non-VUR grouped patients were treated with antibiotic according to culture sensitivity report for 10 days. Meticulous follow-up including fluid management, wound management, drains and catheter management had been taken during post-operative period for all operative patients. Patients were routinely followed up at 2, 6 and $12 \mathrm{Wks}$ after initial visit. During each follow-up fever, dribbling, frequent voiding, urinary stream and color of urine were recorded and urine $\mathrm{R} / \mathrm{M} / \mathrm{E}$, urine $\mathrm{C} / \mathrm{S}$, serum Creatinine, blood urea were done with Ultrasonogram of KUB at $12 \mathrm{Wks}$ in VUR grouped patients. Weight and blood pressure were also recorded in each follow-up.

Data collection and Data analysis

Data was collected from primary source in a predesigned data collection sheet. Data were processed manually and analyzed with the help of SPSS (Statistical package for social sciences) 16.0 for windows. A probability value (p) of $<0.05$ was considered statistically significant. Figure-1 shows the flow chart of the study.

\section{RESULTS}

The age of the patients in this study ranged from 2 months 20 days to 10 years. Majority were $<1$ year in VUR group and 1-5 year in Non-VUR group. The mean \pm SD age was $4.16 \pm 1.94$ years in VUR group and $2.31 \pm 2.84$ in non-VUR group that was statistically significant (Table 1 ). 
Table 1: Age distribution

\begin{tabular}{llllll}
$\begin{array}{llll}\text { Age } \\
\text { (Years) }\end{array}$ & \multicolumn{2}{l}{ VUR $(\mathrm{n}=15)$} & \multicolumn{2}{l}{ Non VUR $(\mathrm{n}=25)$} & P value \\
$<1$ & 7 & $(47)$ & 5 & $(20)$ & \\
$1-5$ & 6 & $(40)$ & 12 & $(48)$ & $>0.10^{\mathrm{ns}}$ \\
$>5$ & 2 & $(13)$ & 8 & $(32)$ & $\left(\mathrm{X}^{2}\right.$ test $)$ \\
Mean \pm SD (Years) & & & & & \\
Unpaired't' test & $4.16 \pm 1.94$ & $2.31 \pm 2.84$ & $<0.05^{*}$ & \\
\hline
\end{tabular}

$\mathrm{ns}=$ Not significant,$*=$ Significant

In this study male was more common in VUR group and female was more common in Non VUR group but statistically not significant. It was found that in VUR group most of the patients $(80 \%)$ were malnourished according to Gomez classification. Nutritional status was normal $(13 \%)$, Grade I malnutrition (26\%), Grade II malnutrition (53\%) and Grade III malnutrition (06\%). Nutritional status was also improving after 12 weeks follow up. In Non VUR group Nutritional status was normal (24\%), Grade I malnutrition (56\%), Grade II malnutrition $(20 \%)$. There was no grade III malnutrition.

UTI patients had varied presentations in this series. Majority of patients presented with more than one symptom. Regular episode of fever was the commonest symptom in both groups of patients followed by failure to thrive and loss of appetite (Table 2).

Table 2: Clinical presentations.

\begin{tabular}{|c|c|c|c|c|c|}
\hline Presentation & & $\mathrm{R}(\mathrm{n}=15)$ & URR & $=25)$ & $\mathrm{P}$ value \\
\hline & NO. & $(\%)$ & No. & $(\%)$ & \\
\hline $\begin{array}{l}\text { Episodes of } \\
\text { unexplained fever }\end{array}$ & 13 & $(86.6)$ & 22 & (88) & $>0.10 \mathrm{~ns}$ \\
\hline Failure to thrive & 12 & $(80.0)$ & 10 & (40) & $>0.10 \mathrm{~ns}$ \\
\hline Loss of appetite & 11 & $(73.0)$ & 16 & (64) & $>0.10 \mathrm{~ns}$ \\
\hline Cloudy urine & 10 & $(66.7)$ & 20 & (80) & $<0.001^{*}$ \\
\hline Urinary frequency & 9 & $(60.0)$ & 14 & (56) & $<0.05^{*}$ \\
\hline $\begin{array}{l}\text { Crying / Burning sensation } \\
\text { during micturition }\end{array}$ & 8 & $(53.3)$ & 15 & $(60)$ & $>0.10 \mathrm{~ns}$ \\
\hline Haematuria & 2 & $(13.0)$ & 0 & (0) & 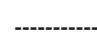 \\
\hline
\end{tabular}

'Z test', * Significant,

ns $=$ Not significant

Raised temperature was the commonest findings in both group followed by Anemia. All patients in this study were normotensive. No palpable flank mass was detected in any patients (Table 3 ).

In the present study, 8 patients with primary VUR (53\%) had associated anomalies (three patients, each had two anomalies). Anorectal malformation was the commonest (27\%) in this series followed by Ectopic kidney (13\%). There was no associated abnormality detected in other group (Table 3 ).
Table 3: Associated anomalies of primary VUR patients $(n=15)$

\begin{tabular}{lcc} 
Associated anomalies & No. of anomalies & Percentage (\%) \\
Anorectal malformation & 4 & 27 \\
Ectopic kidney & 2 & 13 \\
Ureterocele & 2 & 13 \\
Single kidney & 2 & 13 \\
Bilateral Duplex with & & 7 \\
ectopic ureteral opening & 1 & \\
\hline
\end{tabular}

Anemia was the commonest findings in both VUR and nonVUR groups ( $60 \%$ and $44 \%$ respectively). Raised blood urea and Creatinine was found in 3 and 2 patients respectively in primary VUR group. Urine $\mathrm{C} / \mathrm{S}$ examination showed growth of different organisms in $40(100 \%)$ cases. E. Coli was the most common organism isolated in both groups $(46.7 \%$ and $48 \%$ respectively) followed by Pseudomonas, Klebsiella and Acinetobacter.

VCUG was done in all patients in this series. Various grade of VUR was present in 15 patients (38\%). Grade IV reflux (25\%) was more common than other grades. Left sided reflux was more common than right side. Bladder abnormalities were found in VUR patients only. Irregular bladder outline and diverticula were detected in 6 and 2 patients respectively.

Ultrasonogram of KUB region was done in all 40 patients but abnormalities of urinary tract were found in VUR patients only. Hydronephrosis was found in 17 renal units (61\%) and hydroureter in 13 (46\%).

Renogram was done in 9 patients. Gross parenchymal insufficiency was detected in 3 renal units all had grade IV reflux. Scarring was also detected in 3 renal units having grade IV and V reflux (Table 4).

Table 4: Findings of DMSA Renogram $(n=9)$, (Renal units: 16)**

$$
\text { Findings }
$$
No. Of renal Unit Percentage (\%)

\begin{tabular}{lll} 
Reduced cortical function & 6 & 37 \\
Gross parenchymal insufficiency & 3 & 18 \\
Duplex collecting system & 2 & 12 \\
Scarring & 3 & 18 \\
\hline
\end{tabular}

* Done in VUR grade III and above. **Two patients had congenital single renal unit.

Non-VUR grouped patients were treated with antibiotic for 10 days according to urine $\mathrm{C} / \mathrm{S}$ report. Primary VUR grouped patients were treated as follows: bilateral ureteric reimplantation 4, unilateral ureteric reimplantation 2 and nonoperative measures in 8 . One patient died during study period.

Only VUR group patients were followed up. All patients were afebrile and normotensive after 3 months follow up. Frequent voiding continued in 3 patients. There was no urinary tract infection after 12 weeks follow up. 
Table 5: Biochemical and urinary changes $(n=15)$

\begin{tabular}{|c|c|c|c|c|}
\hline Findings & On arrival & $\begin{array}{l}\text { After } \\
1^{\text {st }} \text { follow } \\
\text { up (2Wks) }\end{array}$ & $\begin{array}{l}\text { low-up } \\
2^{\text {nd }} \text { follow } \\
\text { up (6Wks) }\end{array}$ & $\begin{array}{l}3^{\text {rd }} \text { follow } \\
\text { up }(12 \mathrm{Wks})\end{array}$ \\
\hline Documented UTI & 15 & 8 & 6 & 0 \\
\hline Raised B. Urea & 2 & 0 & 0 & 0 \\
\hline Raised S. Creatinine & 1 & 0 & 0 & 0 \\
\hline
\end{tabular}

Table 6: Nutritional status $(\mathrm{n}=15)$

\begin{tabular}{lccc} 
Nutritional status & On arrival & After 12 weeks & P value \\
Normal & 2 & 4 & $>0.50^{\mathrm{ns}}$ \\
Grade I (Mild) & 4 & 7 & $>0.10^{\mathrm{ns}}$ \\
Grade II (Moderate) & 8 & 3 & $>0.10^{\mathrm{ns}}$ \\
Grade III (Severe) & 1 & 0 & ----- \\
\hline 'Z' test & & & $\mathrm{ns}=$ Not significant
\end{tabular}

Hydronephrosis was improved after 12 weeks though this improvement was not statistically significant. But hydroureter improved significantly $(\mathrm{p}<05)$.

\section{DISCUSSION}

The occurrence of VUR decreases with age in our series. This is probably due to the subsequent maturity of VUJ during older age and lowest serum level of IgG and IgA during the first few months of life. This correlates with the study done by Chand et al. (2003) \& Agarwal (2000). In non-VUR group majority were within $1-5$ years ${ }^{11-12}$.

Incidence of VUR was higher in male child $(60 \%)$ in present study. Male predominance was also noted in $<1$ year age group. This male predominance in $<1$ year correlates with the study of Lama et al. (2000) and Huang \&Tsai (1995) ${ }^{13}$. Female sex was predominant in non-VUR group in present study. UTI was more in female child but VUR was more in male child. Hutch (1961) also noted that the incidence of UTI is higher in females than in males ${ }^{14}$.

In this series, $67 \%$ of the patients came from poor income group. Most of the treatment facilities of Dhaka Shishu Hospital are free of cost or with a nominal cost and the lower income group people can avail these facilities. Moreover, this reflects the economic status of population of a country as a whole. In this study, majority of the children (80\%) were suffering from different grades of malnutrition. It was probably due to the most of the patients in present study come from poor family. Disease process may also aggravate the nutritional status.

Only $40 \%$ mothers claimed to have gone through at least one antenatal checkup. But they failed to show any document and no prenatal diagnosis was done in any patient in present study. Greenfield \& Wan (1996) showed that reflux is the most common underlying cause of antenatally detected hydronephrosis ${ }^{15}$. The advantage of identifying children antenatally is that treatment, either prophylaxis or surgery, can be instituted early and further scarring can be prevented.
Patients had varied presentations in this study. Episodes of fever were the predominant clinical scenario in present study in both group of patients, followed by failure to thrive, loss of appetite, cloudy urine, frequent voiding and crying or burning sensation during maturation. Thus patients of both group in our study was present with features of UTI and also voiding dysfunction. These findings correlate with the studies of Fanos and Cataldi (2004), Chand et al. (2003), Agarwal (2000) \& Greenfield and Wan (1996). Agarwal (2000) mentioned that frequency of VUR is directly related to the raised body temperature. In this study raised body temperature was detected in 10 patients $(67 \%)$ of primary VUR group. Only four patients out of 17 male patients were circumcised that is 13 patients $(76.4 \%)$ were uncircumcised. The incidence of UTI in uncircumcised was ten times higher than circumcised boys ${ }^{16}$. All patients in this study were normotensive.

Eight patients of reflux group had been suffering from different associated anomalies like ARM, ectopic kidney, ureterocele, single kidney, and duplex ureter with ectopic ureteral opening. Among them, ARM was the commonest $(27 \%)$. Atala and Keating (2002) in their study also reported ARM to be the commonest associated anomaly with VUR. Kiely and Pena (1998) have shown reflux in 59\% of 38 infants with ARM. No associated abnormalities were found in nonVUR group ${ }^{17}$.

Anaemia was found in both group, almost equally 11 out of 25 (44\%) in non-VUR group and 9 out of $15(60 \%)$ in VUR group. This is probably due to recurrent UTI and poor socioeconomic status in these children. This finding had also reported by Hossain (2002) in his study ${ }^{18}$. Raised blood urea and creatinine was found in 3 and 2 patients respectively in VUR group.

In this study all patients had documented UTI. The most common causative organism was E. Coli (47.5\%). Nuutinen \& Uhari (2001), Svenborg-Eden et al. (1987) and Smellie et al. (1975) also reported that E.coli was the commonest causative organism. $^{19}$

Voiding cystourethrogram was done in all patients in this study. Different grades of VUR were detected in 15 patients (38\%). Despite not having statistical analysis the percentage of VUR in studied population is high (1 in 2.6 patients). VCUG in control group of normal population (without UTI) was not ethically possible as this exposes to radiation hazard apart from expenditure involved. But the result correlates with the results expressed in percentage in studies of Goonasekera and Abeysekera (2003) (12\%-50\%), Agarwal (2000) (25\%$40 \%)$ and Huang \&Tsai (1995) (29\%-50\%). Bladder outline irregularity was present in 6 cases (40\%) and diverticulum in 2 cases $(13 \%)$ of VUR group.

Unilateral reflux $(60 \%)$ was more common than bilateral reflux $(40 \%)$. Left sided reflux $(42.8 \%)$ was more common than right side $(32.1 \%)$. Grade IV reflux $(25 \%)$ more common than other grades. These findings correlate with the study of Lama et al. $(2000)^{20}$.

Ultrasonogram of KUB region was done in all patients in this study but abnormalities was found in VUR patients only. 
Hydronephrosis was in 17 renal units $(61 \%)$ and hydroureter in 13 renal units $(46 \%)$. This disparity between hydronephrosis and hydroureter was probably due to presence of excessive gas in the abdomen during ultrasonogram and milder form of dilatation of ureter, which could not be detected by USG. In this study hydronephrosis was shown by USG in 14 renal units of patients with higher grade of reflux (Grade III and above) that correlate with the study of Aboutaleb et al. (2003). But hydronephrosis was detected in 3 renal unit of patient with grade II VUR. UTI causes dilatation of pelvicalyceal system that was detected in USG.

DMSA renal scan was done for children with VUR grade III and above. In this study renogram was done in 9 patients and the renogram report was analyzed in terms of renal units (16 renal units, as two patient had single renal unit). Renogram showed gross parenchymal insufficiency in 3 renal units (18\%) and scarring in three renal units (18\%). Duplex collecting system was detected in 2 renal units $(18 \%)$. Three renal units that showed scarring had Grade IV and V reflux and below 5 years of age. This finding correlates with the study of Goonasekera \& Abeysekera (2003) ${ }^{4}$.

Among primary reflux group, surgery (Cohen ureteric reimplantation) was done in six patients, eight patients were treated non-operatively and one patient died of renal failure and septicaemia. Bilateral ureteric reimplantation done in four patients, three for bilateral grade IV and V reflux and another for grade III reflux with left sided ureterocele and right sided VUJ obstruction. Unilateral ureteric reimplantation done in two patients, both having single renal unit and grade III reflux. Eight patients were treated non-operatively. Among them one patient had bilateral duplex system with right-sided grade II and left sided grade IV VUR. She underwent left-sided upper pole heminephroureterectomy for duplex system. There after she had been managed conservatively for VUR following operation. Postoperative period of all operative patients were uneventful.
At the time of discharge, the patients were advised to take prophylactic antibiotic, perineal hygiene, taking adequate water, regular bowel habit and to report at the surgical out patient department for follow-up after 2, 6 and 12 weeks. All patients came for follow-up as per schedule. During each follow-up urine $\mathrm{R} / \mathrm{M} / \mathrm{E}$ and $\mathrm{C} / \mathrm{S}$, blood urea, serum creatinine were done with ultrasonogram of KUB region after 12 weeks. Blood pressure was monitored at every follow-up. Features of UTI (fever, urinary stream, cloudy urine, urinary frequency) were also observed in each follow-up.

Non-VUR grouped patients were treated with antibiotic, according to culture-sensitivity report for 10 days and advised circumcision for non-circumcised male patient and also advised to maintain perineal hygiene, taking adequate water, regular bowel habit.

After 12 weeks all patients were afebrile. Urine C/S had not shown any growth of bacteria. There was good urine flow but urinary frequency was persisted in 3 patients. Blood pressure was normal in all patients on admission and after 12 weeks of follow up, though scarring was present in three renal units on admission. Wolfish et al. (1993) postulated that primary uncomplicated VUR, even if associated with renal scars, does not lead to hypertension. ${ }^{21}$ All patients had normal blood urea and creatinine. Nutritional status of the patients was also improved. Ultrasonogram findings showed hydronephrosis improved after 12 weeks follow-up. Overall outcome of management after limited follow up period was good.

\section{CONCLUSION}

UTI especially in male child was associated with VUR in $38 \%$ of this study population. ARM was the common associated anomaly. Renal scarring was noted in higher grade of reflux. UTI should be treated keeping in mind of possibility of VUR \& its damaging consequence on kidney, which may even, be fatal. Patient with ARM with UTI especially intermediate and high variety should be screened for associated VUR before discharge.

\section{DISCLOSURE}

All the authors declared no competing interest. 


\section{REFERENCES}

1. Mahant, S., To, T. and Friedman, J. ' Timing of voiding cystourethrogram in the investigation of urinary tract infections in children', The journal of pediatrics. 2001;(139):568-571.

2. Shortlife,L.M.D., Shinghal,R. and Seto, E.H. 'Pediatric Urinary Tract Infection 'In Pediatric Urology, Gearhart, J.P., Rink, R.C.and Mouriquand, P.D.E., WB Saunders, Philadelphia. 2001;16:237-258.

3. Denes, F.T. and Arap, S. 'Vesicoureteral reflux in children', Journal of Pediatrics (RioJ). 1995;71:183-188.

4. Goonasekera, C.D.A.\& Abeysekera, C.K. 'Vesicoureteric Reflux and Reflux Nephropathy', Indian Journal of Pediatrics. 2003;70:241-249.

5. Godley, M.L. 'Vesicoureteral reflux: Pathophysiology and Experimental Studieds' In Pediatric Urology, Gearhart, J.P., Rink, R.C. and Mouriquand, P.D.E., WB Saunders, Philadelphia. 2001;24:359-381.

6. Smellie, J., Edwards, D., Hunter, N., Normand, I.C.S. and Prescod, N. 'Vesicoureteral reflux and renal scarring', Kidney International. 1975;8:65-72.

7. Sheldon, A.C., Minevich, E. and Wacksman, J. 'Urinary tract infection and vesicoureteral reflux', In Pediatric Surgery. $3^{\text {rd }}$ ed., Ashcraft,K.W., Murphy, J.P., Sharp, R.J., Sigalet, D.L. and Snyder, C.L., Saunders, Philadelphia. 2000;54:706-724.

8. American Academy of Pediatrics. 'Practice parameter: The diagnosis, treatment, and evaluation of the initial urinary tract infection in febrile infants and young children', Pediatrics. 1999;103:843-852.

9. Lebowitz, R.L., Olbing, H., Parkkulainen, K.V., Smellie, J.M. and Tamminen- Mobius, T.E. 'International system of radiographic grading of Vesicoureteral reflux', Pediatric Radiology. 1985;15:105-109.

10. Ritchey, M.L. and Bloom, D. 'Report of the American Academy of Pediatrics Section of Urology Meeting', Pediatric Nephrology. 1995;9:642-646.

11. Chand, D.H., Rhoades, T., Poe, S.A., Kraus, S. and Strife, C.F. 'Incidence and severity of Vesicoureteral reflux in children related to age, gender, race and diagnosis', The Journal of Urology. 2003;170:1548-1550.

12. Agarwal, S. 'Vesicoureteral reflux and urinary tract infections', Current Opinion in Urology. 2000;10:587-592.

13. Huang, F.Y. and Tsai, T.C. 'Resolution of vesicoureteral reflux during medical management in children', Pediatric Nephrology. 1995;9:715-717.

14. Hutch, J.A. 'Theory of maturation of the intravesical ureter', The Journal of Urology. 1961;86:534-538.

15. Greenfield, S.P. and Wan, J. 'Vesicoureteral reflux: Practical aspects of evaluation and management', Pediatric Nephrology. 1996;10:789-794.

16. Fanos, V. and Cataldi, L. 'Antibiotics or sursery for vesicoureteric reflux in children', Lancet. 2004;364:1720-22.

17. Kiely, E.M. and Pena, A. 'Anorectal Malformations' In Pediatric Surgery,O’ Neill, J.A., Rowe, M.I., Grosfeld, J.L., Fonkalsrud, E.W. and Coran, A.G., Mosby, Philadelphia. 1998;95:1425-1460.

18. Hossain, A.Z. January 2002, 'Posterior uretheral valves: Urinary tract changes and management' MS (Pediatrics) thesis, Bangladesh Institute of Child Health, Dhaka.

19. Nuutinen, M. and Uhari, M. 'Recurrence and follow-up after urinary tract infection under the age of 1 year', Pediatric Nephrology. 2001;16:69-72.

20. Lama, G., Russo, M., Rosa, E.D., Mansi, L., Piscitelli, A., Luongo, I. and Salsano, M.E. 'Primary vesicoureteric reflux and renal damage in the first year of life', Pediatric Nephrology. 2000;15:205-210.

21. Wolfish, N.M., Delbrouck, N.F., Shanon, A., Matzinger, M.A., Stenstrom, R. and McLaine, P.N. 'Prevalence of hypertension in children with primary vesicoureteral reflux', The Journal of Pediatrics. 1993;123:559-563. 